\title{
Marie Diekmann/Lea Welsch \\ Die neuen Rechten und der Streit um Meinungsfreiheit
}

\section{Einleitung}

Das Terrain der Meinungsfreiheit ist seit jeher politisch wie juristisch umkämpft. Seit einiger Zeit bemängeln Mitglieder und Anhänger der AfD, der Neuen Rechten und Rechtskonservative, ${ }^{1}$ die Meinungsfreiheit sei bedroht und werde zugunsten eines linken Meinungskonsenses beschränkt. Der Vorsitzende der Deutschen Polizeigewerkschaft Rainer Wendt sieht, nachdem gegen seinen Auftritt an der Universität Frankfurt protestiert und er ausgeladen wurde, "Meinungsfreiheit und [...] Wahrheit" als „Opfer". 2 Die AfD bringt regelmäßig Rosa Luxemburgs Aphorismus, Freiheit sei immer die Freiheit der Andersdenkenden, für sich und gegen ihre Kritiker*innen in Anschlag. ${ }^{3}$ Und Professor*innen fürchten angesichts des „Konformitätszwangs“ der angeblichen „Political Correctness" um die universitäre Streitkultur. ${ }^{4}$ Dabei vermischen sich juristisch-dogmatische, politische und kulturelle Fragestellungen zu einer diffusen Gesamtdiagnose, die bis ins linke Lager hinein Anklang findet. Der Versuch, die Thesen der (universitären) Ver-

1 Ohne völlig übergehen zu wollen, dass es sich hierbei um unterschiedliche Akteure mit unterschiedlicher ideologischer Ausrichtung handelt, ist es aus unserer Sicht zulässig, hier ein wenig zu pauschalisieren - und das nicht nur aus Platzgründen. Denn es gibt mannigfaltige ideologische Schnittstellen sowie persönliche Verbindungen innerhalb des rechten Lagers. Dem Rechtskonservatismus, wie er etwa von der „Werteunion“ vertreten wird, kommt zudem eine wichtige Vermittlungsfunktion zu. Gerade der Kampf gegen „linke Meinungsdiktatur“ und die Verbreitung sogenannter „migrationskritischer" Meinungen sind zudem diskursive Projekte, die unterschiedliche Akteure verbindet, was aber nicht bedeuten soll, dass etwa Rechtsradikalismus und Konservatismus inhaltlich gleichgesetzt oder die gesamte AfD mit der Neuen Rechten in eins gesetzt werden.

2 Mitteilung der Deutschen Polizeigewerkschaft vom 26. Oktober 2017, https://www.dpolg.de/aktue lles/news/das-opfer-ist-die-meinungsfreiheit/.

3 So den Social Media-Auftritten der AfD zu entnehmen: https://de-de.facebook.com/wirhierinlbg/p hotos/freiheit-nur-f\% C3\% Bcr-die-anh\% C3\% A4nger-der-regierung-nur-f\% $\mathrm{C} 3 \% \mathrm{Bcr}$-mitglieder-e iner-partei-m\%C3\%B6ge/889268631133124/ oder auch der rechten Internet-Zeitschrift „Die Freie Welt", Die Andersdenkenden, https://www.freiewelt.net/blog/die-andersdenkenden-10043057/, oder als Extrembeispiel, die Vereinnahmung Rosa Luxemburgs durch Social Media-Beiträge rechter Politiker, https://twitter.com/albertobagnai/status/906175541438550017).

4 So etwa der Deutsche Hochschullehrerverband: Resolution des 67. DHV-Tages in München, Zur Streit- und Debattenkultur an Universitäten, https://www.hochschulverband.de/fileadmin/redaktio n/download/pdf/resolutionen/ResolutionPoliticalCorrectness-Endfassung.pdf.; Besonders irritierend ist der Beitrag von Sarah Diefenbach und Daniel Ullrich, der die zentrale These des Buches, die Meinungsfreiheit sei massiv bedroht, nicht nur nicht belegt, sondern rechte Verschwörungstheorien als Fakten präsentiert: Es war doch gut gemeint. Wie Political Correctness unsere freiheitliche Gesellschaft zerstört, Riva/München 2017. 
teidiger*innen der Meinungsfreiheit nachzuvollziehen, scheitert jedoch meistens, da es an fundierten Begründungen mangelt.

Betrachtet man die Rechtsprechung zur Meinungsfreiheit, ist von einer meinungsfreiheitsfeindlichen Tendenz zuungunsten rechter Positionen nichts zu erkennen. Im Gegenteil: Spektakuläre Einzelentscheidungen lassen zumindest vereinzelt eine Offenheit nach rechtsaußen auch innerhalb der Richter*innenschaft vermuten. ${ }^{5}$

Im Folgenden wollen wir die Dogmatik der Meinungsfreiheit speziell im Hinblick auf ihren Umgang mit rechten Meinungen rekapitulieren. Das Grundgesetz ist dabei, in der Interpretation des Bundesverfassungsgerichts, selbst verfassungsfeindlichen Meinungen gegenüber tolerant. In der Auseinandersetzung mit rechtsradikalen Meinungen stellte die Verfassungsgerichtsbarkeit jedoch klar, dass sie diese zwar formal zulasse, dabei aber auf gesellschaftlichen Widerspruch setze.

Im Weiteren werden wir darstellen, dass gerade diejenigen, die sich rechten Meinungen entgegenstellen, nun als Zensor*innen und damit als Feinde der Meinungsfreiheit erscheinen. Die Meinungsfreiheit fungiert hier bei genauerer Betrachtung meist nicht als juristisches Konzept, sondern als diskursive Strategie zur Abwehr von Protest. Währenddessen versucht die Gesetzgebung, zumindest in den sozialen Netzwerken Einfluss zu gewinnen und gegen Hate Speech vorzugehen. Nicht zuletzt die Kritiken daran machen jedoch deutlich, wie schwierig und problematisch die Regulierung von Meinungsäußerungen bleibt. Damit stellt sich einmal mehr die Frage, ob das Recht ein angemessenes Mittel sein kann, um mit den Auswüchsen eines gesamtgesellschaftlichen Rechtsrucks umzugehen.

\section{Die Meinungsfreibeit in der Rechtsprechung}

\subsection{Demokratie und Meinungsfreiheit}

Die historischen Wurzeln der Meinungsfreiheit reichen zurück zur Französischen Revolution, in der durch die Bestimmung der freien Meinungsäußerung als zentrales Menschen- und Bürgerrecht die Kritik politischer Herrschaft sichergestellt werden sollte. Die Meinungsfreiheit ist damit Zeugnis und Ausdruck des politischen Kampfes gegen autoritäre Obrigkeitsstrukturen ohne demokratische Meinungsvielfalt. Je mehr die Freiheit, eigene Ansichten öffentlich zu äußern, gesetzlichen oder faktischen Beschränkungen unterliegt, desto schlechter scheint es um die Demokratie bestellt zu sein. ${ }^{6}$ Entsprechend wird dem in Art. 5 Abs. 1 Satz 1 GG verbürgten Recht, „seine Meinung in Wort, Schrift und Bild frei zu äußern und zu verbreiten“, auch von Jurist*innen große Bedeutung zugemessen. Dies äußert sich vor allem in einer herausgehobenen Stellung innerhalb der Grundrechtearchitektur. Die Gerichte haben den Anwendungsbereich der Meinungsfreiheit im Rahmen seiner Ausdifferenzierung auch zuungunsten konkurrierender Verfassungsgüter, wie beispielsweise dem allgemeinen Persönlichkeitsrecht, stetig erweitert. Im

5 Vgl. hierzu etwa das Interview von Malte Engler von der Neuen Richtervereinigung: https://www.h essenschau.de/gesellschaft/richter-ueber-giessener-npd-urteil-20-seiten-lang-faschistische-slogans-g ekloppt,urteil-npd-giessen-100.html.

6 Siehe dazu mit pointiertem Überblick: Vincent Klausmann, Meinungsfreiheit und Rechtsextremismus, Baden-Baden 2019, 84; mit Verweis auf die historische Situation in Deutschland siehe Bethge, in: Sachs (Hrsg.), GG-Kommentar, Art. 5 Rn. 5 a. 
Konfliktfall gilt es, im Zweifel die für die Meinungsfreiheit günstigere Auslegung zu wählen.

Die Dogmatik der Meinungsfreiheit wurde maßgeblich von der Rechtsprechung des Bundesverfassungsgerichts bestimmt. In der Lüth-Entscheidung von 1958 heißt es dazu:

„Das Grundrecht auf freie Meinungsäußerung ist als unmittelbarster Ausdruck der menschlichen Persönlichkeit in der Gesellschaft eines der vornehmsten Menschenrechte überhaupt [...]. Für eine freiheitlich-demokratische Staatsordnung ist es schlechthin konstituierend, denn es ermöglicht erst die ständige geistige Auseinandersetzung, den Kampf der Meinungen, der ibr Lebenselement ist (BVerfGE 5, 85 [205]). Es ist in gewissem Sinn die Grundlage jeder Freibeit überhaupt, the matrix, the indispensable condition of nearly every other form of freedom (Cardozo) “"?

Nach diesem Verständnis des Verfassungsgerichts verknüpfen sich mit der Meinungsfreiheit nicht nur die Freiheit, sich zu äußern im Sinne eines Abwehrrechts, sondern auch persönlichkeitsbildende Aspekte einer demokratischen Bürgerlichkeit.

\subsection{Schutzbereich der Meinungsfreiheit}

Der Schutzbereich der Meinungsfreiheit ist, verglichen mit der besonders weitgehenden US-amerikanischen Redefreiheit, zumindest dem Wortlaut nach, enger gefasst. Nicht geschützt sind falsche Tatsachenbehauptungen. Leugnung des Holocaust fällt damit ebenso wie sogenannte „Fake News“ grundsätzlich nicht in den Schutzbereich. Zur Abgrenzung von geschützten polemischen Werturteilen und nicht mehr geschützter Schmähkritik, bei der nicht mehr die sachliche Auseinandersetzung, sondern die Diffamierung einer Person im Vordergrund steht, ${ }^{8}$ stellt das Bundesverfassungsgericht auf den situativen Kontext des Einzelfalls ab. ${ }^{9}$

Gerade die Kontextualisierung scheint Gerichten aber bisweilen Probleme zu bereiten, und die Interpretation nimmt mitunter absurde Züge an. Große Aufmerksamkeit erlangte etwa kürzlich die Entscheidung des Berliner Landgerichts, in der die Bezeichnungen der Grünen-Abgeordneten Renate Künast u.a. als „Drecks Fotze“ und „Schlampe“ als Kritik mit Sachbezug gewertet wurden. ${ }^{10}$ Der Beschluss erfuhr viel Kritik und wurde daraufhin im Rechtsmittelverfahren teilweise abgeändert. ${ }^{11}$ Nicht zuletzt wegen der teilweisen Aufhebung kann der Beschluss daher wohl nicht als exemplarisch für die gesamte Rechtsprechung zur Meinungsfreiheit gelten. Dennoch zeigt er besonders drastisch, wie die Rechtsprechung speziell rassistische und misogyne Reden zur sachlichen Kritik verklärt. Denn das Gericht begründet den Sachbezug der sexistischen Beleidigungen ausge-

7 Zitiert nach Thomas Vesting, Soziale Geltungsansprüche in fragmentierten Öffentlichkeiten. Zur neueren Diskussion über das Verhältnis von Ehrenschutz und Meinungsfreiheit, AöR 122 (1997), 337-371 (351); BVerfGE 7, 198 (209).

8 Vgl. BVerfGE 82, 272 (283 f.).

9 Vgl. BVerfGE 93, 266 (294).

10 LG Berlin, Beschluss vom 9. September 2019-27 AR 17/19 (Rn, 38, 35).

11 Interview und Kommentar zum Urteil mit Anna-Lena von Hodenberg, https://netzpolitik.org/20 20/dieses-urteil-ist-ein-gutes-zeichen/. 
rechnet mit der „Sexualisiertheit“ des Themas. ${ }^{12}$ Eine etwas ähnliche Argumentation findet sich auch in einer Entscheidung des Amtsgerichts Frankfurt, in der das Gericht meint, das Beharren auf die öffentliche Benennung einer Mieterin als „Fräulein“ sei nicht beleidigend, da sie früher als normal galt. ${ }^{13}$ Während das Gericht den beklagten Vermieter*innen nicht zumuten will, ihren „älteren Wortschatz und entsprechende moralische Wertungen“ zumindest äußerlich aus Rücksichtnahme zu ändern, empfiehlt es der als „Fräulein“ angesprochenen Mieterin ein wenig mehr „Selbstironie“, was auch daran liegen mag, dass das Gericht in dem Begriff euphemistisch lediglich eine „Verniedlichung“ sieht. ${ }^{14}$ Über die Verwendung des „N-Wortes“ räsoniert das Landesverfassungsgericht Mecklenburg-Vorpommern, ${ }^{15}$ der Begriff würde zwar „nach heutigem Sprachgebrauch in der Regel als abwertend verstanden“, ausgerechnet das Beharren auf der Begrifflichkeit in Kenntnis der beleidigenden Bedeutung werten die Richteræinnen dann aber als „inhaltliche Auseinandersetzung“. Sowohl in der „N-Wort“- als auch in der „Fräulein“-Entscheidung fällt auf, dass die Gerichte ausgerechnet das Beharren auf diskriminierenden Begriffen für zulässig halten. In beiden Fällen geht es gerade nicht darum, dass Begriffe mit Diskriminierungsgeschichte per se und immer unzulässig sein sollen. Beide Fälle landeten erst vor Gericht, weil die Beteiligten vehement auf den diskriminierenden Begriffen bestanden. Gerade diejenigen, die überall „sprachpolizeiliche“ Maßnahmen „politischer Korrektheit“ fürchten, sollten sich also beruhigen können. In keinem der Fälle geht es um sprachliche Ausrutscher, einen misslungenen sexistischen oder rassistischen Witz oder ähnliches. Es geht vielmehr um Fälle, in denen bewusst und wiederholt auf diskriminierender Sprache bestanden wird. Und selbst dann, das zeigen besagte Fälle, hat man in Deutschland gute Chancen auf Verständnis der entscheidenden Richter*innen zu stoBen.

In all diesen Entscheidungen wird denjenigen, die bereits strukturell von Diskriminierung betroffen sind, zugemutet, Ansprachen, die ihre beleidigende Kraft gerade aus dieser Diskriminierungsgeschichte ziehen, hinzunehmen. Die Rechtsprechung scheint dazu zu neigen, historische Diskriminierungen als Kontext zu werten, der die Verwendung diskriminierender Begriffe legitimiert.

\subsection{Die Schranken der Meinungsfreiheit}

Die Diskussion über die Grenzen der Meinungsfreiheit entzündete sich bereits in der Vergangenheit anhand rechtsradikaler öffentlicher Auftritte. Insbesondere über nationalsozialistische Propaganda und speziell die Leugnung des Holocausts wurde gestritten, sowie darüber, welche Anforderungen an die Allgemeinheit des Gesetzes zu stellen sei-

12 Hintergrund der Debatte ist ein Zwischenruf von Renate Künast, den diese im Zusammenhang der Pädophilie-Debatte 1986 im Berliner Abgeordnetenhaus getätigt hatte. In einem Bericht der "Welt" von 2015 wurde dieser Zwischenruf aufgegriffen und dahingehend interpretiert, dass Künast sich damals für die Straffreiheit für Sex mit Kindern ausgesprochen hätte. Künast wies diese Interpretation zurück. Die von ihr gerichtlich geltend gemachten Hass-Postings bewegen sich im Kontext dieser medialen Auseinandersetzung.

13 AG Frankfurt, Urteil vom 27.6.2019 - 29 C 1220/19 (46).

14 Garonne Bezjak/Eva Kocher, Man wird ja wohl noch Fräulein sagen dürfen? Zum Urteil des AG Frankfurt am Main vom 27. Juni 2019, Az.: 29 C 1220/19 (46), myops 38 (2019), 25-30.

15 LVerfG MV, Urteil vom 19.12.2019 - LVerfG 1/19, BeckRS 2019, 32968. 
en. ${ }^{16}$ Dabei stellte sich die Frage, ob Gesetzgebung, Behörden und Gerichte auf faschistische Meinungskundgaben und öffentliche Aufmärsche mit Verboten reagieren durften oder ob diesem die Meinungsfreiheit entgegenstünde. Mit den Reformen des $\$ 130$ StGB (der Jahre 1994 und zuletzt 2005) pönalisierte der Gesetzgeber einerseits die sogenannte Auschwitzlüge und statuierte darüber hinaus ein Verbot, die nationalsozialistische Gewalt- und Willkürherrschaft zu billigen, zu verherrlichen oder zu rechtfertigen. Mit diesen Strafrechtsreformen reagierte die Politik auf die zunehmende Sichtbarkeit von Neonazis im öffentlichen Raum. ${ }^{17}$

Das Bundesverfassungsgericht befand den 2005 eingeführten Absatz 4 des $\ 130$ StGB als mit dem verfassungsrechtlichen Allgemeinheitsgrundsatz der Schranken unvereinbar, erklärte ihn aber dennoch in seinem Wunsiedel-Beschluss von 2009 für verfassungsgemäß. ${ }^{18}$ Die Ausnahme vom Grundsatz der Allgemeinheit der Schranken sei „insoweit die Reaktion des Gesetzgebers auf konkrete politische, als besonders gefährlich beurteilte Auffassungen im öffentlichen Meinungskampf." 19 In seiner historischen Einmaligkeit hätten die Grauen des Nationalsozialismus insofern prägenden Charakter, als dass ausnahmsweise die Äußerung einer „bestimmten Meinung, nämlich der Gutheißung der nationalsozialistischen Gewalt- und Willkürherrschaft” verboten werden könne. ${ }^{20}$ Die identitätsbildende Abgrenzung des Grundgesetzes zum historischen Nationalsozialismus beinhalte jedoch im Kern „kein allgemeines antinationalsozialistisches Grundprinzip“.

Das Bundesverfassungsgericht wählte damit eine von vielen Jurist*innen als widersprüchlich kritisierte ${ }^{21}$ Kompromisslösung, wonach dem Grundgesetz eine eng begrenzte antifaschistische Bedeutung zukommt - allerdings ausschließlich bezogen auf den historischen Nationalsozialismus. Deshalb kann seine positive Bewertung strafrechtlich sanktioniert werden. Das Grundgesetz schützt aber grundsätzlich, nach Rechtsprechung des Bundesverfassungsgerichts, alle anderen Formen der Meinungsäußerung - inklusive faschistischer Meinungen.

16 Diese Frage, was unter allgemeinem Gesetz zu verstehen ist, ist Gegenstand verschiedensten dogmatischen Streites, der bis zu Diskussionen über den Vorläufer der Meinungsfreiheit des Art. 5 Abs. 1 Satz 1 GG in der Weimarer Reichsverfassung zurückreicht. Vgl. Matthias Jestaedt, $\mathbb{} 102$

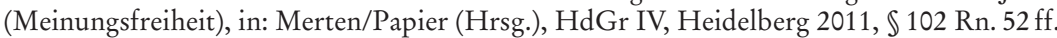

17 LK-StGB $/ \operatorname{Krau} \beta, \mathbb{S} 130$, Rn. 17; weiterführend zur kritischen Reflexion im deutschen Justizkontext: Monika Frommel, Fremdenfeindliche Gewalt, Polizei und Strafjustiz, KJ 1994, $323 \mathrm{ff}$.

18 Siehe zu dieser Einordnung und zum stellenweise ambivalenten Charakter geschichtsbezogener Strafrechtsvorschriften: Felix Hanschmann, Geschichtsbezogene Strafvorschriften als Herausforderung der Meinungsfreiheit, KJ 2013, 307-324.

19 BVerfGE 124, 300 (326).

20 BVerfGE 124, 300 (326f.).

21 Uwe Volkmann, Die Geistesfreiheit und der Ungeist - Der Wunsiedel-Beschluss des BVerfG, NJW 2010, 417 ff.; Stefan Martini, Diskriminierung (Rechts-) Extremer Meinungen nach Art. 5 II GG, Jahrbuch des öffentlichen Rechts der Gegenwart, Band 59 (2011), 279-303. 


\section{Der liberale Einwand der Standpunktneutralität}

Dieses im Ergebnis sehr weite Verständnis der Meinungsfreiheit, das explizit selbst faschistische Meinungen schützt, ist im Vergleich zur Rechtsprechung des US-amerikanischen Supreme Courts eher restriktiv. ${ }^{22}$

Die juristische Literatur formuliert einhellig Kritik am Wunsiedel-Beschluss, kann sich jedoch nicht dazu durchringen, die Entscheidung als falsch, weil restriktiv, zu bezeichnen. Dies scheint die historische Dimension von Wunsiedel zu gebieten. Die Freiheitsaffirmationen und Anrufungen demokratischer Diskurse, an der ausnahmslos alle beteiligt werden sollen, berufen sich jedoch auf die Forderung der Neutralität des Rechts gegenüber jeder öffentlichen Äußerung, egal aus welchem politischen Lager sie kommt. Die Demokratie beweist sich demnach gerade in ihrer Toleranz gegenüber ihren Feinden. Vorherrschend ist ein betont liberales Verständnis der Meinungsfreiheit. Dabei sind bei genauerem Hinsehen zwei Arten liberaler Argumentation analytisch zu unterscheiden.

\subsection{Vermeintliche Liberalität}

Einige liberale Verteidiger*innen der Meinungsfreiheit gehen in ihrer Neutralitätsforderung sehr weit. So äußerte Winfried Brugger bereits vor dem Wunsiedel-Beschluss im Hinblick auf den Ausschluss der Auschwitzlüge, dass selbst erwiesenermaßen falsche Tatsachenbehauptungen einen guten Beitrag zur öffentlichen Debatte leisten könnten. Der „erwartbare Effekt von Holocaust-Leugnungen“ sei im Ergebnis „Gewinn für die schreckliche - Wahrheit“, „[b]ei der Ausschwitz-Leugnung“ führe „die Unwahrheit wahrscheinlich zu mehr Gutem als Schlechtem für das Gemeinwesen. “23. Das Bundesverfassungsgericht aber praktiziere, indem es die Holocaust-Leugnung aus dem Schutzbereich herausnehme, im Sinne des „Zeitgeistes“ „Meinungsdiskriminierung“, indem es "Pazifismus“ für „akzeptabler" halte als „Rechtsradikalismus“. ${ }^{24}$ Der Beitrag zeigt, wie vermeintlich liberale Positionen bisweilen selbst Wertungen von Meinungen vornehmen. Brugger geht dabei soweit, dass er, wenn auch als Frage getarnt, selbst rechten Positionen Raum gibt, indem er die gängige antisemitische Behauptung anbringt, Jüd*innen würden den Holocaust nutzen, um sich politische und finanzielle Vorteile zu verschaffen.

„Und weiß man genau, daß einige Juden oder auch sonstige Gruppen den Holocaust nicht für politischen und finanziellen Ausgleich benutzen?" 25

Er verrät an dieser Stelle, dass er nicht nur meint, der positive Gehalt der Holocaustleugnung bestünde in der öffentlichen Empörung und anschließenden Wahrheitsfindung, sondern dass er ihr einen eigenen Wahrheitsgehalt beimisst, da sie (womöglich) auf eine

22 Einige Autor*innen sehen in der Entscheidung jedoch eine Annäherung an die US-amerikanische Dogmatik, Matthias Hong, Das Sonderrechtsverbot als Verbot der Standpunktdiskriminierung Der Wunsiedel-Beschluss und aktuelle versammlungsgesetzliche Regelungen und Vorhaben, DVBl. 2010,1267-1276 (1269f.).

23 Winfried Brugger, Verbot oder Schutz von Haßrede?, AöR 128 (2003), 372-411 (398).

24 Ebd., 403.

25 Ebd., 407. 
perfide Strategie von Opfern des Holocausts, sich diesen zu Nutze zu machen, hinweisen könne. ${ }^{26}$

Auch wenn dies wohl einen extremen Fall der Neutralitätsthese darstellt, so wird doch ein Grundproblem sichtbar: Auch die vermeintlich neutrale Ausrichtung des Rechts der Meinungsfreiheit kommt nicht ohne Wertungen aus, und manche sich als liberal verstehende Verteidigung der Meinungsfreiheit entlarvt sich bei genauerer Betrachtung als verkappte Sympathiebekundung für rechte Meinungen, die eben nicht nur für die Neutralität des Rechts gegenüber allen Meinungen plädiert, sondern dabei implizit eine Aufwertung rechter Meinungen vornimmt.

\subsection{Vertrauen in den demokratischen Diskurs}

Anders verhält es sich allerdings mit einer anderen Spielart des liberalen Einwands, die nicht darauf angewiesen ist, alle Positionen gleichermaßen zu wertvollen Beiträgen zum demokratischen Diskurs zu verklären. In dieser Variante verlangt die liberale Kritik, dass auch undemokratische und diskriminierende Positionen zulässig sein müssen. Sie seien dann im Diskurs selbst als undemokratisch zu entlarven, nicht aber rechtlich zu sanktionieren. Diese Position findet sich etwa in der Rechtsprechung des Bundesverfassungsgerichts, das klarstellt, dass auch gefährliche, unbegründete Meinungen geschützt seien. Das gelte auch für verfassungsfeindliche Positionen, denn im Kern stehe das Grundgesetz zwar für spezifische Werte, erzwinge dahingehend jedoch keine Loyalität. ${ }^{27}$ Zentrale Prämisse dieser liberalen Konzeption der Meinungsfreiheit ist das Vertrauen auf die Rationalität des freien demokratischen Diskurses. So meint etwa das Bundesverfassungsgericht im Wunsiedel-Beschluss:

„Das Grundgesetz gewäbrt Meinungsfreibeit im Vertrauen auf die Kraft der freien öffentlichen Auseinandersetzung vielmehr grundsätzlich auch den Feinden der Freibeit. " 28

Dabei stellt sich die Frage, worin dieses Vertrauen angesichts unzähliger empirischer Gegenbeispiele eigentlich gründet. Selbst der an dem Wunsiedel-Beschluss beteiligte Verfassungsrichter Johannes Masing bezweifelt die Rationalität öffentlicher Debatten. Demnach begründet sich die Garantie der Meinungsfreiheit zwar in einem a priori bestehenden Vertrauen hinsichtlich dessen, dass rechtlich gewährte Freiheiten „schließlich zum Guten“ führten, gleichzeitig betont Masing die realen Gefahren von Meinungen, die auf die Erschütterung fundamentaler demokratischer Prämissen abzielten, und weist auf die „irrationalen Effekte medial vermittelter Kommunikation“ hin. ${ }^{29}$ Sinnvoll kann es sich daher nur um eine kontrafaktische normative Prämisse handeln. Demnach ist der demokratische Diskurs keine vorgängige soziale Tatsache, auf der die Meinungsfreiheit beruht. Die Ermöglichung eines tatsächlich freien öffentlichen Diskurses ist vielmehr Aufgabe

26 Die naheliegende Antwort auf diese Suggestivfrage lautet schlicht: Ja, manche Opfer des Holocausts fordern materielle oder immaterielle Anerkennung des ihnen angetanen Leids. Dass Brugger hier ein Problem andeutet, lässt Ungutes vermuten.

27 BVerfGE 124, 300 (320).

28 Ebd.

29 Johannes Masing, Meinungsfreiheit und Schutz der verfassungsrechtlichen Ordnung, JZ 2012, 385-392 (385). 
der Demokratie. Die Meinungsfreiheit sichert damit nur das subjektive Recht, sich am Diskurs zu beteiligen, unabhängig von der Qualität des Diskursbeitrags. Sie sichert aber nicht den freien demokratischen Diskurs selbst ab, was sich auch daran ablesen lässt, dass es neben der Meinungsfreiheit andere auf diskursive Teilhabe abzielende Grundrechte in der Verfassung gibt. Die Meinungsfreiheit ist Teil des einheitlichen Kommunikationsgrundrechtes, in dem sowohl Presse- als auch Informations-, Kunst- und Wissenschaftsfreiheit angesiedelt sind. Zudem schützt die Verfassung mit der Versammlungs- und Vereinigungsfreiheit auch kollektive Meinungskundgaben und Interessenverfolgung. ${ }^{30}$

\section{Dogmatik vs. Realität: zur (Un)möglicbkeit freier Kommunikation}

Dem öffentlichen Diskurs kommt demnach die Rolle zu, das, was die Meinungsfreiheit sichert, zu moderieren. Rechtsradikale Meinungen können frei geäußert werden, es ist Aufgabe der Zivilgesellschaft, ihnen zu widersprechen und sie zu marginalisieren. Das gelang bisher mehr oder weniger. Spätestens aber seitdem Vertreter*innen der Neuen Rechten in Parlamenten und Talkshows sitzen, hat sich der öffentliche Diskurs zugunsten rechter Positionen verschoben.

\subsection{Diskursive Strategien: Doppeldeutigkeit als Programm}

Die Akteure der Neuen Rechten haben sich längst mit der strafrechtlichen und gesellschaftlichen Ächtung des Nationalsozialismus arrangiert. Sie definieren sich gerade über eine doppeldeutige Abgrenzung zum historischen Nationalsozialismus. Sie rücken ihre Gegner*innen in die Nähe des Faschismus und beziehen sich gleichzeitig inhaltlich und semantisch auf ihn. Bereits in den 1970er Jahren haben Akteure der Neuen Rechten Konzepte wie den „Ethnopluralismus“ entwickelt, der ohne direkte Bezüge zu biologistischen Rassenkonzepten auskommt, dennoch aber wesentliche rassentheoretische Elemente beibehält. Dabei zeigen die Neuen Rechten durchaus eine gewisse Pluralität und ideologische Flexibilität ${ }^{31}$ und sind somit sehr viel anschlussfähiger als „traditionelle“ Rechtsradikale, wie sie die NPD repräsentiert. Ein Grund hierfür ist wohl, dass die Akteure der AfD genau darauf achten, die Grenzen zu Straftatbeständen nicht zu überschreiten. Sie leugnen den Holocaust nicht, sie relativieren ihn. Sie beziehen sich nicht auf die vermeintliche „Rasse“ von Menschen, sondern auf deren Herkunft und Kultur. Damit umgehen sie die rechtliche Ächtung und können die Meinungsfreiheit als grundgesetzliche Garantie für sich beanspruchen. So bekennt sich die AfD in ihrem Grundsatzprogramm zwar zu einer „offenen und freien Gesellschaft“, in der Menschen „unabhängig von ihrer Herkunft und Religion“ friedlich zusammenleben. ${ }^{32}$ Diesem formellen Bekenntnis korrespondieren allerdings keine Inhalte, im Gegenteil: Menschen mit nichtdeutscher Herkunft werden durchweg negativ adressiert und der „ethnisch-kulturelle

30 Frotscher/Pieroth, Verfassungsgeschichte, München 2018, Rn. 815.

31 Helmut Kellershohn, Die Neue Rechte: Wo sie herkommt, was sie will, wohin sie geht, Duisburger Institut für Sprach- und Sozialforschung 2017, https://www.diss-duisburg.de/2017/07/helmutkellershohn-die-neue-rechte/.

32 Programm für Deutschland. Das Grundsatzprogramm der Alternative für Deutschland, 46. 
[...] Wandel der Bevölkerungsstruktur“ zu einem der drängendsten Probleme erklärt. ${ }^{33}$ Damit ersetzt der Begriff der Kultur den Rassebegriff funktional, d.h., er rechtfertigt ebenjene Politiken, die vormals mit Verweisen auf „rassische“ Unterschiede begründet wurden. Gleichzeitig wird so dauerhaft eine Leerstelle offengehalten, die jeder Zeit mit traditionellen rassistischen Vorstellungen aufgeladen werden kann, wie es etwa Björn Höcke immer wieder tut. Besonders deutlich wurde das etwa in seinem bekannt gewordenen Vortrag zur Asylpolitik vom November 2015, in dem er in klassisch rassistischer Manier einen „afrikanischen Ausbreitungstyp" von einem "europäischen Platzhaltertyp" unterscheidet. $^{34}$

Der Literaturwissenschaftler Heinrich Detering zeigt exemplarisch anhand einiger bekannter Redebeiträge wichtiger AfD-Funktionäre auf, wie die Partei mit Mehrdeutigkeiten, widersprüchlichen Aussagen und Andeutungen auf ein völkisch konnotiertes Kollektivsubjekt "Deutsches Volk“ in Abgrenzung $\mathrm{zu}$ einem biologistisch konnotierten "Anderen“ referiert und zum zentralen Ausgangspunkt politischen Handelns macht. ${ }^{35}$ Diese rhetorische Strategie ist allerdings - so offensichtlich und banal sie sein mag - mit einem sich auf einzelne Wörter und Begriffe fokussierenden Blick nur schwer zu fassen. Erst in ihrem Kontext werden das Ausmaß und die Radikalität der AfD-Rhetorik ${ }^{36}$ erkennbar. Es handelt sich bei den Aussagen der AfD keineswegs um polemische Kritik, sondern - nimmt man die AfD beim Wort, so wie Detering es macht - um radikal-antidemokratische und gegen die Grundrechteordnung gerichtete Aussagen. Die Schwierigkeiten, dies zu begreifen und begreifbar zu machen, die Detering im öffentlichen Diskurs ausmacht, zeigen sich auch im Recht. Die explizite Zensur des Rechts scheint zu einfach $\mathrm{zu}$ lesen als dass sie eine effektive Regulierung leisten könnte, ${ }^{37}$ und das Recht tut sich schwer damit, auf derivative diskursive Strategien zu reagieren. Stattdessen scheint es in Form der Meinungsfreiheit auf Seiten der Demokratie- und Grundrechteverächter*innen zu stehen. Dabei zeigt sich immer wieder ein diskursiver Effekt, den die Verteidiger*innen eines liberalen Verständnisses der Meinungsfreiheit zu übersehen scheinen: Die Meinungsfreiheit verleiht demokratiefeindlichen Reden Legitimation. Sie erscheinen somit nicht als nur demokratiefeindlich, aber dennoch zulässig, sondern darüber hinaus als inhaltlich wertvoll.

Denn das strikt liberale Verständnis der Meinungsfreiheit neigt zu einem rein formalen Demokratieverständnis, das Meinungsäußerungen nur ihrer Form nach beurteilen, aber nicht zwischen demokratischen und undemokratischen Inhalten unterscheiden kann. Dabei schleicht sich eine Art Bedeutungsübertragung von der Form auf den Inhalt ein: Meinungen, die in einer Demokratie hingenommen werden müssen, sich aber gegen zentrale

33 Ebd., 82.

34 Vgl. Steven Geyer, “Lebensbejahender afrikanischer Ausbreitungstyp”: AfD-Spitze rügt Höckes Rassenkunde-Referat als parteischädigend, 14.12.2015, https://www.berliner-zeitung.de/politikgesellschaft/lebensbejahender-afrikanischer-ausbreitungstyp-afd-spitze-ruegt-hoeckes-rassenkunde-referat-als-parteischaedigend-li.30732.

35 Heinrich Detering, Was heißt hier »wir«? Zur Rhetorik der parlamentarischen Rechten, Ditzingen 2019.

36 Diese Rhetorik ist keine rein diskursive Strategie, sondern eingebunden in ein - bei genauerem Hinsehen - ebenso radikales politisches Programm, vgl. Marie Diekmann, „Hin zu einer nationalen Bevölkerungspolitik“ - Was das Programm der AfD für die Grundrechte bedeuten könnte, in: Grundrechte-Report 2019, Frankfurt/M. 2019, 135-139.

37 Vgl. Judith Butler, Hass spricht. Zur Politik des Performativen, Berlin 1998, 185. 
normative Grundannahmen von Demokratie und Menschenrechten richten, werden auch als inhaltlich demokratisch wahrgenommen. Die Meinungsfreiheit fungiert dann als demokratisches Label für undemokratische Meinungen. ${ }^{38}$ Diese „talismanische Kraft ${ }^{\text {“ } 39}$ hat keine rechtliche Wirkung, immunisiert aber gegen Kritik. Der Vorwurf, in der eigenen Meinungsfreiheit ${ }^{40}$ durch Kritiker\%innen eingeschränkt zu sein, lenkt den Vorwurf unzulässiger Rede von den Redner*innen auf die Kritiker*innen, die dann nicht ebenfalls als Redner*innen mit Meinungsfreiheit, sondern als Zensor*innen erscheinen. Auch hier sind es auffällig oft rechte Akteure, die sich von Kritiker*innen zensiert fühlen. Absurd wird es spätestens, wenn etwa offene Briefe, die Rassismus anprangern, als Zensur diffamiert werden oder Redner*innen, denen ohnehin viel öffentliche Aufmerksamkeit zukommt, sich von sehr viel weniger profilierten Kritiker*innen zensiert fühlen. ${ }^{41}$

\subsection{Strukturelle Begünstigung rechter Meinungen}

Zudem hat sich mit dem Internet und den sozialen Medien der öffentliche Diskurs neu strukturiert. Was zunächst nach einer Demokratisierung der öffentlichen Meinung aussah, wurde schnell auch zum Problem für den demokratischen Diskurs. Mit Facebook und Co. ist eine digitale Infrastruktur entstanden, die jeder Person Zugang zum öffentlichen Meinungsaustausch gewährt, ohne dass die die traditionellen Medien kennzeichnenden Gatekeeper passiert werden müssten.

Allerdings wird diese Infrastruktur keineswegs notwendig zu demokratischen Zwecken genutzt. Zwar verbreiten sich über die sozialen Medien tatsächlich Meinungen und Inhalte, die außerhalb der Agenda traditioneller Medien liegen, und ermöglichen somit eine neue Inhalts- und Meinungsvielfalt. Nur ein Teil dieser Inhalte beruht aber auf Fakten, und nur ein Teil dieser Meinungen steht in Einklang mit basalen demokratischen Werten und den Grundrechten. Zahlreiche Studien belegen die strukturelle Überlegenheit rechtsradikaler Inhalte in den sozialen Medien. ${ }^{42}$ Soziale Medien sind demnach nicht einfach als neutrale Plattformen zu verstehen, sondern als mediale Orte, die aufgrund ihrer spezifischen Strukturierung manipulative Bedrohungsszenarien, die einen wesentlichen Teil rechter Propaganda ausmachen, besonders gut verbreiten. Das macht deutlich, dass der demokratische Diskurs sich nicht von selbst einstellt, sondern angewiesen ist auf eine entsprechende Infrastruktur sowie auf diejenigen, die undemokratische Meinungen als solche benennen und ausgrenzen.

38 Der US-Amerikaner Stanley Fish meint dazu: "[...] the label 'free speech' is the one you want your favorites to wear."; Stanley Fish, There is no such thing as free speech, and it's a good thing, too, Oxford (Univ. Press) 1994, 102.

39 Ebd. 111.

40 An Universitäten wird sie in der Regel gemeinsam mit der Wissenschaftsfreiheit in Anschlag gebracht, die an dieser Stelle ähnlich funktioniert.

41 So z. B. im Fall Wendt, hierzu: Katharina Hoppe/Darja Klingenberg/Vanessa Eileen Thompson/ Felix Trautmann/Alexander Vorbrugg, Worüber wir reden, wenn wir mit jemandem nicht reden wollen, movements, Vol.4, Issue 1 /2018, S.167-177; zum Fall Baberowski: Andreas FischerLescano, Rechte und Rechtswissenschaft, VerfBlog, 2019/9/29:,https://verfassungsblog.de/rechteund-rechtswissenschaft/,DOI:https://doi.org/10.17176/20190929-232454-0.

42 M.w.N. Maik Fielitz/Holger Marcks, Digital Fascism: Challenges for the Open Society in Times of Social Media, Berkeley Center for Right-Wing Studies, Working Paper Series, Juli 2019, online: https://escholarship.org/uc/item/87w5c5gp. 


\subsection{Hate Speech}

Ein altbekanntes Problem der Dogmatik der Meinungsfreiheit wird zudem im digitalen Raum besonders virulent: die Abgrenzung von „rein geistig“ bleibenden und handlungsinduzierenden Meinungsäußerungen. Nach der Rechtsprechung des Bundesverfassungsgerichts genießt die "rein geistige Sphäre des Für-richtig-Haltens" "43 vorbehaltlosen grundrechtlichen Schutz. Erst Äußerungen, welche „die Schwelle zur individualisierbaren, konkret fassbaren Gefahr einer Rechtsverletzung“ überschreiten und „über die Überzeugungsbildung hinaus mittelbar auf Realwirkungen angelegt sind“, können unterbunden werden. Beispielhaft nennt das Gericht hierfür: „Appelle [...] zum Rechtsbruch, aggressive [...] Emotionalisierungen oder d[ie] Herabsetzung von Hemmschwellen“, welche „rechtsgutgefährdende Folgen unmittelbar auslösen können“. ${ }^{44}$

Abgesehen davon, dass die Vorstellung eines „rein geistigen“ Sprechens bei genauerer Betrachtung absurd und die Grenzziehung zu handlungsinduzierender Rede konstruiert anmutet, wird diese Grenzziehung insbesondere, wenn sich aggressives Sprechen gegen bereits von Diskriminierung betroffene Menschen richtet, prekär. Sehr viel stärker als im nationalstaatlichen Kontext scheint innerhalb internationaler Menschenrechtsregime die Überzeugung präsent zu sein, dass Meinungsäußerungen, wenn sie auf die Herabwürdigung von Individuen oder Gruppen abzielen und somit das menschenrechtliche Prinzip der Gleichheit angreifen, immer auch aus rechtlicher Perspektive problematisch sind, da sie einen Missbrauch der freien Rede bedeuten, wenn ihr vornehmlicher Inhalt in der Missachtung Anderer und ihrer Rechte besteht. Rassistisches, oder allgemein, aggressives Sprechen wird dabei unter dem Begriff der Hate Speech verhandelt. Der aus der amerikanischen Debatte über die Redefreiheit stammende Begriff spielt seit langem eine wichtige Rolle in der Dogmatik der internationalen Menschenrechte. ${ }^{45}$ Damit fallen etwa rassistische Äußerungen nicht automatisch aus dem Schutzbereich. Sie sind dogmatisch jedoch anders gerahmt, da bereits auf Ebene des geschützten Verhaltens der normative Zusammenhang der Menschenrechte unter dem Begriff Hate Speech thematisiert wird. Darüber, weshalb dem Gebrauch der freien Rede zur Verbreitung von Hass gegen ohnehin schon von Diskriminierung betroffene Gruppen im internationalen Recht mehr Bedeutung innerhalb der Dogmatik zukommt, ließe sich einiges sagen. Jedenfalls scheint im internationalen Kontext der Zusammenhang von rassistischer Gewalt (bis hin zu Genoziden) und rassistischer Rhetorik präsenter als im nationalstaatlichen. ${ }^{46}$

43 BVerfGE 124, 300 (330).

44 Ebd.

45 Siehe hierzu: Christoph Apostel, Hate Speech - zur Relevanz und den Folgen eines Massenphänomens, KriPoZ 2019, 287 ff. Sehr übersichtlich außerdem: Factsheed des EGMR zum Thema Hate Speech, Juni 2020 https://www.echr.coe.int/Documents/FS_Hate_speech_ENG.pdf, und des Wissenschaftlichen Dienstes des Bundestags: Sachstand zu Hassrede (hate speech) und HolocaustLeugnung in der menschenrechtlichen Spruchpraxis, März 2015: https://www.bundestag.de/resou rce/blob/485798/13870af2cbd422605e56121a9821a7fo/WD-2-055-15-pdf-data.pdf.

46 Die hier implizierte Vermutung ist, dass aufgrund des stärkeren Fokus auf Genozide und Gewalt gegen Minderheiten in den internationalen Menschenrechtsregimen die Rechte anders zueinander ins Verhältnis gesetzt werden als im nationalen Kontext. Zwar führt nicht jede rassistische öffentliche Rhetorik zu Gewalt gegen Minderheiten. Dennoch werden Genozide immer von rassistischer Rhetorik eingeleitet und begleitet. Dadurch ist der Zusammenhang von bestimmten Formen freier Rede und der Missachtung der Rechte anderer sehr viel präsenter. Gleichzeitig - und das scheint uns ein wichtiger Unterschied zu Deutschland, dessen Verfassung ebenfalls in Abgrenzung auf 
Mit dem Anstieg rechter Rhetorik und Gewalt rückt der Begriff der Hate Speech bzw. Hassrede aber auch in Deutschland in den Fokus. So verweist die Begründung zum im Oktober 2017 in Kraft getretenen Netzwerkdurchsetzungsgesetz (NetzDG) etwa explizit auf den Anstieg von Hassrede und Fake News in den sozialen Netzwerken. ${ }^{47}$ Zwar gibt es eine Vielzahl (teils berechtigter) kritischer Einwände gegen das NetzDG, das unter anderem private Plattformbetreiber*innen in die Position versetzt $\mathrm{zu}$ entscheiden, welche Äußerungen zulässig sein sollen und welche nicht. ${ }^{48}$ Das Gesetz ist aber dennoch auch als Versuch zu sehen, den demokratischen Diskurs abzusichern. ${ }^{49}$

\section{Der Rechtsformeinwand: Reinszenierung und Willkür}

Gerade im Hinblick auf eine strategische Nutzung der Meinungsfreiheit, den Freiheitsraum minoritärer oder besonders häufig von diskriminierenden Äußerungen betroffener Menschen zu beeinträchtigen, scheint eine Regulierung der freien Rede naheliegend.

Dagegen spricht jedoch, dass, selbst wenn durchaus zwischen demokratischen und undemokratischen Meinungen bzw. zwischen aggressivem Sprechen und Hassrede unterschieden werden kann, diese Grenzziehung immer aufs Neue und im konkreten Fall erfolgen muss. Rechtliche Verbote bleiben dabei einerseits unvollständig, weil sie ihr Ziel, einen demokratischen Diskurs zu ermöglichen, nie vollständig erreichen können, und drohen zudem selbst undemokratisch zu werden, da die Grenzziehung immer ein Stück willkürlich bleibt.

Einen solchen grundsätzlichen Einwand gegen rechtsförmige Regulierungen der Redefreiheit formuliert Judith Butler. Rechtliche Verbote verfehlen demnach ihre Zielsetzung, indem sie das Zensierte reinszenieren, dabei aber gleichzeitig immer unvollständig bleiben. ${ }^{50}$ Butler macht so auf ein wichtiges Argument aufmerksam, das liberale Verteidiger*innen der Meinungsfreiheit auf ihrer Seite haben: Selbst wenn es möglich ist, zwischen in einer Demokratie noch zulässigen und nicht mehr zulässigen Meinungen zu unterscheiden, bleibt diese Grenzziehung im Grunde unsicher. Gerade Äußerungen, die in diesem Grenzbereich zu verorten sind, erlangen spätestens, wenn sie gerichtlich verhandelt werden, erst recht öffentliche Aufmerksamkeit. Die „N-Wort“-Entscheidung des Mecklenburgischen Verfassungsgerichts wiederholt den umstrittenen Begriff nicht nur mehr als 20-mal, sondern erklärt gerade die bewusst-provokative Verwendung zur zulässigen Kritik. Die Gerichtsentscheidung wiederholt damit den von der AfD im Parlament inszenierten Rassismus und steigert ihn vielleicht sogar. Nun ist die hier exemplarisch gewählte Entscheidung eine, die anders hätte getroffen und vor allem anders hätte begründet werden können. Das Problem der Reinszenierung stellt sich aber selbst dann, wenn das Recht gezielt antirassistisch ausgerichtet wird. Sogar das Antidiskriminierungsrecht

einen Genozid bezogen ist - haben die internationalen Richter*innen mehr Distanz zu den nationalen Akteuren, die Hass propagieren.

47 BT-Drs. 18/12356 (16.5.2017).

48 Siehe zu diesem und weiteren Einwänden Kristina Bautze, Verantwortung im Netz - Anmerkungen zum Netzwerkdurchsetzungsgesetz, KJ 2019, 203-212 (208 ff.).

49 So beschreibt es auch der gemeinnützige Verein hateaid, der sich beratend für die Opfer von Hasskriminalität einsetzt. Siehe: hateaid.org.

50 Butler, Hass spricht (Fn. 37), $182 \mathrm{ff}$. 
wiederholt zumindest implizit essentialistische Festschreibungen. ${ }^{51}$ Dabei tendiert der juristische Diskurs dazu, den politischen $\mathrm{zu}$ vereinnahmen. ${ }^{52}$ Demnach wird es immer schwieriger, Aussagen, die rechtlich vielleicht noch zulässig, aber trotzdem rassistisch sind, auch als solche kenntlich zu machen. Das Recht steht für Butler zudem nicht automatisch auf Seiten der von Hassrede und Diskriminierung Betroffenen - im Gegenteil: Verbote laufen laut Butler immer auch Gefahr, sich gegen Minderheiten zu richten. Stattdessen setzt Butler auf die subversive Resignifikation und Aneignung verletzender bzw. diskriminierender Sprache. ${ }^{53}$ Es gibt, so könnte man mit Butler argumentieren, eigentlich keinen Grund, dem Recht und denjenigen, die es auslegen, mehr zuzutrauen, als dem gesellschaftlichen Diskurs und denjenigen, die daran teilnehmen. Und es gibt keinen Grund, die Verantwortung, gegen Diskriminierung einzustehen, allein ins Recht zu verlagern. Widerspruch und Praktiken der Umdeutung brauchen sich nicht auf das Recht zu berufen. Butler mag dabei die Möglichkeiten der diskursiven Aneignung über- und die Möglichkeiten des Rechts unterschätzen, ${ }^{54}$ sie verdeutlicht aber, wie schwierig die Regulierung von Meinungsäußerungen tatsächlich ist. Und das gilt nicht nur für das Recht, sondern auch für öffentliche und universitäre Diskurse. Rassistische Begriffe verschwinden dadurch ebenso wenig wie rassistische Ideologien. Dies entbindet aber nicht von der, gerade auch durch die Meinungsfreiheit auferlegten Aufgabe, antidemokratischen Meinungen entgegenzutreten. Der Protest gegen Rechte ist nicht mit Zensur gleichzusetzen und selbst von der Meinungsfreiheit geschützt. Und die rechtliche Gleichbehandlung aller Meinungen bedeutet nicht, dass rechte Meinungen einen Anspruch darauf hätten, unwidersprochen verbreitet $\mathrm{zu}$ werden.

51 Vgl. hierzu ausführlich: Wendy Brown, Die Paradoxien der Rechte ertragen, in: Christoph Menke/ Francesca Raimondi (Hrsg.), Die Revolution der Menschenrechte. Grundlegende Texte zu einem neuen Begriff des Politischen, Berlin 2011, 454-473.

52 Butler, Hass spricht (Fn. 37), 76.

53 Butler, Hass spricht (Fn. 37), $140 \mathrm{ff}$.

54 Vgl. kritisch zu Butler: Nikita Dhawan, Meinungsfreiheit, Hassrede und die Politiken der Zensur, Feministische Studien 2/18, 322- 334 (327 ff.). 